\title{
Theory of spike correlations: a formal description of input and output correlations in spiking neurons Rubén Moreno-Bote*1 and Néstor Parga ${ }^{2}$
}

\author{
Address: ${ }^{1}$ Center for Neural Science, New York University, USA and ${ }^{2}$ Universidad Autónoma de Madrid, Madrid, Spain \\ Email: Rubén Moreno-Bote* - rmoreno@cns.nyu.edu \\ * Corresponding author
}

from Sixteenth Annual Computational Neuroscience Meeting: CNS*2007

Toronto, Canada. 7-12 July 2007

Published: 6 July 2007

BMC Neuroscience 2007, 8(Suppl 2):P43 doi: I0.I I86/I47I-2202-8-S2-P43

() 2007 Moreno-Bote and Parga; licensee BioMed Central Ltd.

Spike correlations between neurons are ubiquitous in cortex, but their role is at present not understood. Here we describe the firing response of a leaky integrate-and-fire neuron when it receives a temporarily correlated input generated by pre-synaptic correlated neuronal populations. Input correlations are characterized in terms of the firing rates, Fano factors, correlation coefficients and correlation time scale of the neurons driving the target neuron. It has been shown [1] that the sum of the pre-synaptic spike trains cannot be well described by a Poisson process. In fact, the total current has a non-trivial two-point correlation function described by two main parameters: the correlation time scale (how precise the input correlations are in time), and the correlation magnitude (how strong they are). Therefore, the total current generated by the input spike trains cannot be approximated by a white noise Gaussian process in the diffusion limit. Instead, the total current is replaced by a colored Gaussian process with the same mean and two-point correlation function, leading to the formulation of the problem in terms of a Fokker-Planck equation. Solutions of the output firing rate are found in the limit of short and long correlations time scales. The solutions described here expand and improve our previous results [1] by presenting new analytical expressions for the output firing rate for general IF neurons, extending the validity of the results for arbitrarily large correlation magnitude, and by describing the differential effect of correlations on the mean driven or noise dominated firing regimes. In addition, we also study the correlated output spike trains of two neurons receiving independent as well as common sources of Gaussian noise. This formalism [2] describes analytically the Fano factor of the output spike count, the output auto-correlation function and output cross-correlation function of the spiking response of a pair of neurons. These results open the door to the study of spike correlations in neuronal networks and their role in neural processing and information transmission.

\section{References}

I. Moreno R, de la Rocha J, Renart A, Parga N: Response of spiking neurons to correlated inputs. Physical Review Letters 2002, 89(28):288I0I

2. Moreno-Bote R, Parga N: Auto and cross-correlograms for the spike response of LIF neurons with slow synapses. Physical Review Letters 2006, 96:028101. 